Check for updates

Cite this: RSC Adv., 2017, 7, 50935

\title{
Density functional theory study on the boron and phosphorus doping of germanium quantum dots
}

\author{
Zhenyi Ni, (D) Hui Jia, Xiaodong $\mathrm{Pi}^{*}$ and Deren Yang \\ Doping is a crucial way of tuning the properties of semiconductor quantum dots (QDs). As one type of \\ important semiconductor QDs, germanium (Ge) QDs have been recently doped with boron (B) and \\ phosphorus (P) through a gas-phase synthesis approach successfully. However, theoretical \\ understanding about the structural and electronic properties of doped Ge QDs remains rather limited. \\ Here we investigate the doping of Ge QDs with $B$ and $P$ in the framework of density functional theory. \\ The formation energies and electronic structures of singularly B- or P-doped Ge QDs and B/P-codoped \\ Ge QDs are systematically studied. It is found that both B and P prefer the near-surface region of Ge \\ QDs. The electronic structures of Ge QDs can be effectively tuned by B and P doping. The B/P codoping \\ may facilitate the incorporation of $B$ and $P$ into Ge QDs, resulting in the further modification of the \\ electronic structures of Ge QDs.
}

Received 15th August 2017

DOI: $10.1039 / \mathrm{c} 7 \mathrm{ra0} 9008 \mathrm{k}$

rsc.li/rsc-advances

(DFT) has been extensively employed to study the doping of a variety of semiconductor QDs such as the group IV counterpart of Ge QDs-silicon (Si) QDs. ${ }^{43-49}$ With the development of the experimental study on the doping of Ge QDs, it is now imperative that theoretical work such as DFT investigation ought to be carried out to explain the experimental findings on the doping of Ge QDs and predict the properties of doped Ge QDs.

In this work, we construct hydrogen (H)-passivated Ge-QD models with a diameter of $1.4 \mathrm{~nm}$ to investigate the $\mathrm{B}$ and $\mathrm{P}$ doping of Ge QDs within the framework of DFT. The formation energies of dopants in Ge QDs are calculated. The electronic structures of singularly B- or P-doped Ge QDs and B/P-codoped Ge QDs are systematically studied. It is found that B or P is the most likely incorporated in the near-surface region of Ge QDs. B is more likely doped than P. B introduces deep energy levels in the bandgap of a Ge QD whether it is inside or in the nearsurface region of the QD. In contrast, $\mathrm{P}$ gives rise to deep energy levels only when it is incorporated inside a Ge QD. It is interesting that the deep energy levels induced by $\mathrm{P}$ are deeper than those induced by $\mathrm{B}$. The $\mathrm{B} / \mathrm{P}$ codoping may promote the incorporation of $\mathrm{B}$ and $\mathrm{P}$ into Ge QDs. The Ge-QD bandgap is effectively reduced by the $\mathrm{B} / \mathrm{P}$ codoping.

\section{Method}

The optimization of structures and the calculation of total energies are performed at $0 \mathrm{~K}$ by using the all-electron DFT modeling package DMol3. ${ }^{46,50,51}$ The Becke-Lee-Yang-Parr (BLYP) exchange-correlation functional at the generalized gradient approximation (GGA) level is used. Double numerical basis sets augmented with p-polarization functions (DNP basis

State Key Laboratory of Silicon Materials, School of Materials Science and Engineering, Zhejiang University, Hangzhou, Zhejiang 310027, China. E-mail: xdpi@zju.edu.cn 
sets) are employed as the atomic orbital basis functions. To ensure accurate calculation, a high self-consistent field (SCF) convergence threshold of $10^{-6}$ and a large orbital cutoff of $4.6 \AA$ are employed. The maximum energy change and forces on all of the atoms in the optimized structures are less than $10^{-5} \mathrm{Ha}$ and $0.002 \mathrm{Ha} \AA^{-1}$, respectively. To prevent the optimizer from taking unreasonable steps, the maximum allowed change of any cartesian coordinate during the structural relaxation is less than $0.3 \AA$. The highest occupied molecular orbital (HOMO) and lowest unoccupied molecular orbital (LUMO) are sampled on a grid with a spacing of $0.2 \AA$.

\section{Results and discussion}

\section{B or P doping}

Fig. 1 shows the model of a $1.4 \mathrm{~nm} \mathrm{Ge} \mathrm{QD,} \mathrm{which} \mathrm{is} \mathrm{constructed}$ by cutting out a spherical portion in an optimized bulk Ge model. $\mathrm{H}$ atoms are used to passivate the dangling bonds of $\mathrm{Ge}$ atoms at the surface of the QD. Three types of hydrides $(\mathrm{GeH}$, $\mathrm{GeH}_{2}$ and $\mathrm{GeH}_{3}$ ) are at the QD surface, consistent with experimental observation. ${ }^{42}$ The obtained Ge QD is in the form of $\mathrm{Ge}_{71} \mathrm{H}_{84}$. When a substitutional $\mathrm{B}(\mathrm{P})$ atom moves along the path of $1 \rightarrow 2 \rightarrow 3 \rightarrow 4$ in a Ge QD (from the center toward the surface of a Ge QD), it is denoted as B1 (P1), B2 (P2), B3 (P3) or $\mathrm{B} 4(\mathrm{P} 4)$. When a $\mathrm{B}(\mathrm{P})$ atom replaces a Ge atom that is originally connected to one, two or three $\mathrm{H}$ atoms at the surface, it is denoted as $\mathrm{B}_{\alpha}\left(\mathrm{P}_{\alpha}\right), \mathrm{B}_{\beta}\left(\mathrm{P}_{\beta}\right)$ or $\mathrm{B}_{\gamma}\left(\mathrm{P}_{\gamma}\right)$, respectively. $\mathrm{B}_{\alpha}\left(\mathrm{P}_{\alpha}\right), \mathrm{B}_{\beta}\left(\mathrm{P}_{\beta}\right)$ and $\mathrm{B}_{\gamma}\left(\mathrm{P}_{\gamma}\right)$ are passivated by zero (zero), one (one) and zero (two) $\mathrm{H}$ atoms, respectively. Therefore, no dangling bonds are associated with B or P. We should point out that defects like dangling bonds often exist at the surface of as-synthesized QD-

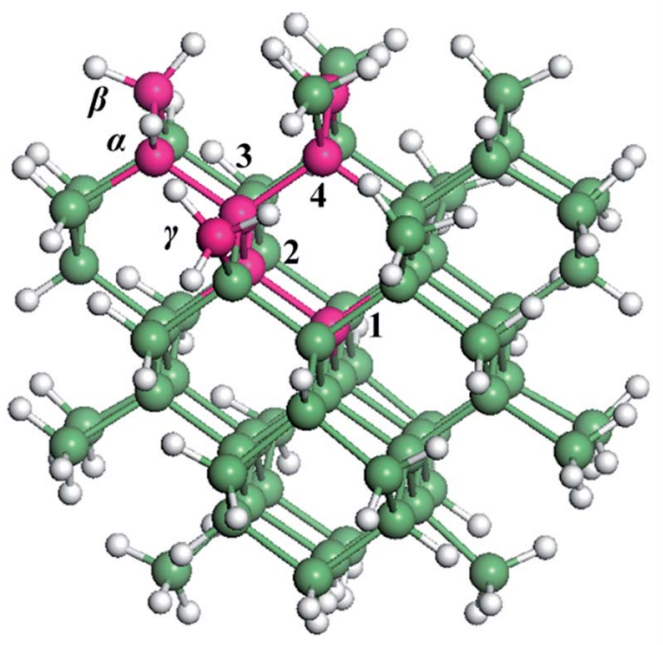

Fig. 1 Model of a B (P)-doped Ge QD with a diameter of $\sim 1.4 \mathrm{~nm}$. Ge and $\mathrm{H}$ atoms are denoted by green and grey balls, respectively. $\mathrm{A}$ substitutional $B(P)$ atom is denoted by a pink ball. B1 (P1), B2 (P2), B3 $(P 3)$ and $B 4(P 4)$ result from moving a substitutional $B(P)$ atom from the center to the surface of the QD along the path of $1 \rightarrow 2 \rightarrow 3 \rightarrow 4$. When a $B(P)$ atom replaces a Ge atom that is originally connected to one, two or three $\mathrm{H}$ atoms at the surface, it is denoted as $\mathrm{B}_{\alpha}\left(\mathrm{P}_{\alpha}\right), \mathrm{B}_{\beta}\left(\mathrm{P}_{\beta}\right)$ or $\mathrm{B}_{\gamma}\left(\mathrm{P}_{\gamma}\right)$, respectively. materials including Ge QDs. ${ }^{26,41}$ By carrying out appropriate surface modification and annealing processes, we may obtain defect-free Ge QDs, akin to that for Si $\mathrm{QDs}^{52,53}$ and twodimensional materials. ${ }^{54,55}$

We calculated the formation energy $\left(E_{\mathrm{f}}\right)$ of a B or P atom in Ge QD by using ${ }^{56,57}$

$$
\begin{aligned}
E_{\mathrm{f}}= & E\left(\mathrm{Ge}_{x^{\prime}} \mathrm{H}_{y^{\prime}} \mathrm{X}\right)-E\left(\mathrm{Ge}_{x} \mathrm{H}_{y}\right)-\mu_{\mathrm{X}} \\
& -\left(x^{\prime}-x\right) \mu_{\mathrm{Ge}}-\left(y^{\prime}-y\right) \mu_{\mathrm{H}},
\end{aligned}
$$

where $\mathrm{X}$ represents $\mathrm{B}$ or $\mathrm{P}, E\left(\mathrm{Ge}_{x} \mathrm{H}_{y}\right)$ and $E\left(\mathrm{Ge}_{x^{\prime}} \mathrm{H}_{y^{\prime}} \mathrm{X}\right)$ are the total energy of a Ge QD before and after the incorporation of a $\mathrm{B}(\mathrm{P})$ atom, respectively. $x\left(x^{\prime}\right)$ and $y\left(y^{\prime}\right)$ are the numbers of Ge and and $\mathrm{H}$ atoms, respectively. $\mu_{\mathrm{Ge}}, \mu_{\mathrm{H}}$ and $\mu_{\mathrm{X}}$ are the chemical potentials of $\mathrm{Ge}, \mathrm{H}$ and $\mathrm{B}(\mathrm{P})$, respectively. Since the relative order of $E_{\mathrm{f}}$ for B or P in all these configurations is not affected by the choices of chemical potentials in a wide range given the linear relationship between $E_{\mathrm{f}}$ and each chemical potential, $\mu_{\mathrm{Ge}}$, $\mu_{\mathrm{H}}, \mu_{\mathrm{B}}$ and $\mu_{\mathrm{P}}$ are set equal to the total energies of atomic Ge in bulk $\mathrm{Ge}$, atomic $\mathrm{H}$ in hydrogen gas, atomic $\mathrm{B}$ in $\mathrm{B}_{12}$ and atomic $\mathrm{P}$ in black phosphorus, respectively. ${ }^{\mathbf{4 3 , 4 4 , 5 8}}$

The calculated $E_{\mathrm{f}}$ for B or P in a Ge QD is shown in Fig. 2. It is seen that the values of $E_{\mathrm{f}}$ for B doping are smaller than those for $\mathrm{P}$ doping in all the configurations, indicating that $\mathrm{B}$ is more likely to be incorporated into a Ge QD than $\mathrm{P}$ in term of thermodynamics. This is in contrast with the doping of $\mathrm{B}$ and $\mathrm{P}$ in H-passivated Si QDs where on average P exhibits a lower $E_{\mathrm{f}}$ than B. ${ }^{59}$ For both B and P doping, the values of $E_{\mathrm{f}}$ at the surface of the QD ( $\alpha, \beta$ and $\gamma)$ are smaller than those inside the QD (1, 2, 3 and 4), validating a preference of the near-surface region for $\mathrm{B}$ and $\mathrm{P}$. The calculated results are in good agreement with experimental observations carried out on the doping of Ge QDs with $\mathrm{B}$ and $\mathrm{P} .{ }^{41,42}$ As a $\mathrm{B}$ or $\mathrm{P}$ atom moves from the center to the sub-surface of the Ge QD (i.e., from $1 \rightarrow 4$ ), the $E_{\mathrm{f}}$ shows a trend of decrease. Similar results have been obtained by B- or P-doped Si QD. ${ }^{43,44,59}$ This is mainly due to the self-purification nature of QDs for impurities. We should mention that the lattice constant of the B- or P-doped Ge QD is basically equal to that of undoped Ge QD. This may because that the current value of the dopant concentration of $1.4 \%$ is too small to significantly affect the

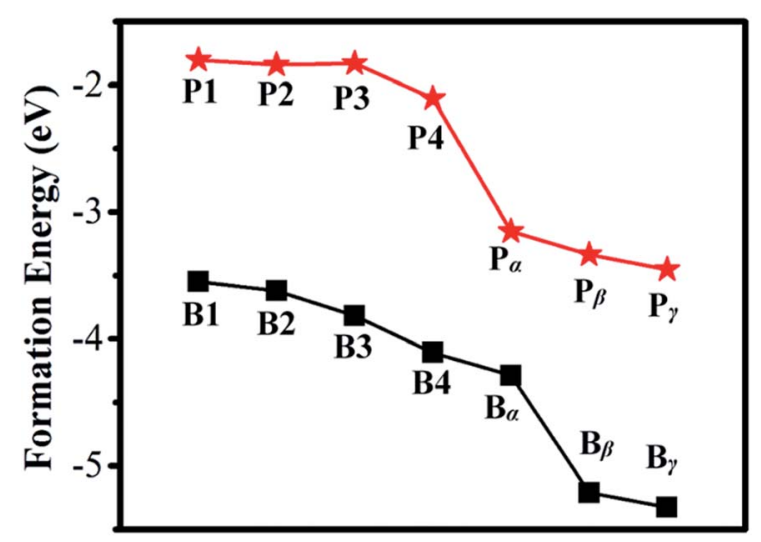

Fig. 2 Formation energy of $\mathrm{B}$ or $\mathrm{P}$ in a Ge QD. The solid lines are used to guide the eye. 
lattice constant of the Ge QD. With the increase of the concentration of the dopant, we may expect a decrease of the lattice constant of the Ge QD according to the Vegard's law, similar to what has been observed in B-doped Si QDs ${ }^{60}$ and carbon- and germanium-doped silicene. ${ }^{61}$

Fig. 3 presents the energy-level diagrams for undoped, B- and P-doped Ge QDs. It is seen that an undoped Ge QD with a diameter of $1.4 \mathrm{~nm}$ shows a bandgap of $2.39 \mathrm{eV}$, which is larger than that of bulk $\mathrm{Ge}$, consistent with the quantum confinement effect. ${ }^{62}$ Similar to carbon- and germanium-doped silicene, ${ }^{61} \mathrm{Ge}$ QDs doped with B or P in different configurations show different band structures. For B-doped Ge QDs, the bandgaps of the QDs are hardly affected $(<5 \%)$ while deep energy levels are introduced in the bandgap (Fig. 3(a)). This is akin to H-passivated Si QDs when they are doped with B via firstprinciples calculations. ${ }^{44}$

When a B atom is in the configurations of B1, B2, B3 or B4, deep energy levels are close to the valence band with a small spin splitting, verifying a p-type characteristic of B-doped Ge QD. This is mainly due to the B-doping-induced extra hole in a Ge QD. In these cases, the electron densities of the HOMO and LUMO (here the HOMO and LUMO are deep energy levels) are
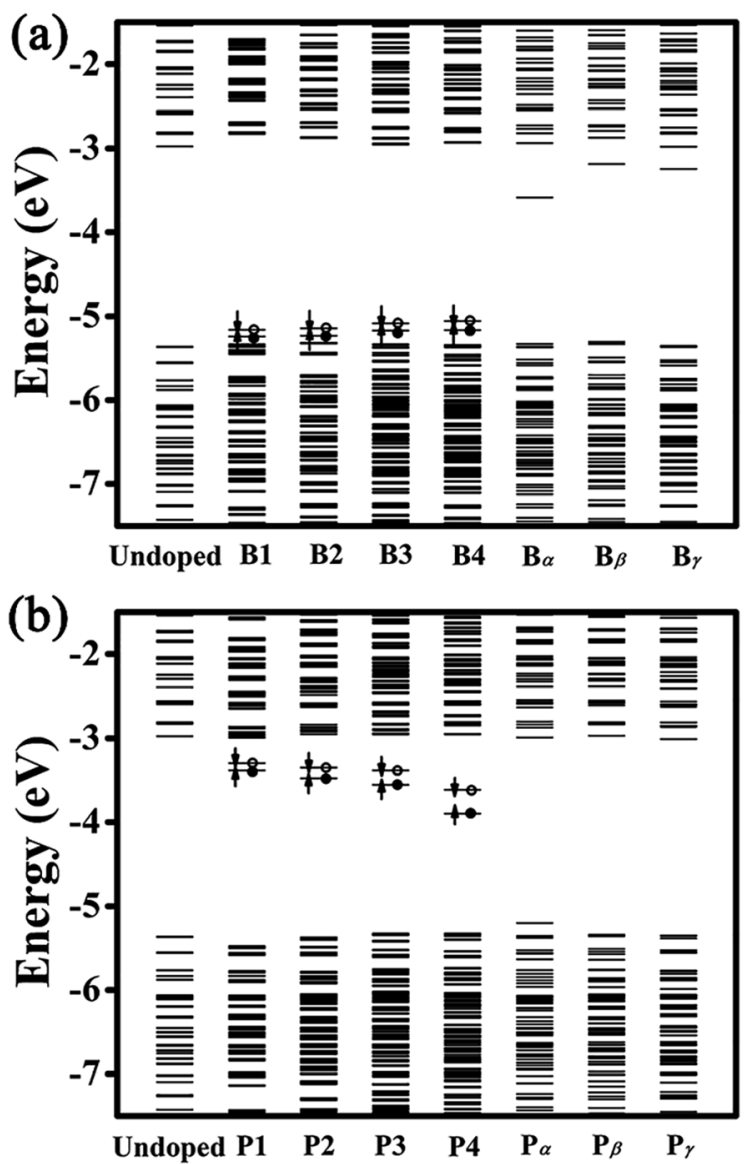

Fig. 3 Energy-level diagrams for undoped, (a) B-doped and (b) Pdoped Ge QDs at the ground state. Filled (empty) circles indicate that energy levels are occupied (unoccupied) by electrons. Spin-up (spindown) states of defect energy levels induced by a B or P atom are indicated by up (down) arrows. mainly located at the B and neighboring Ge atoms with similar distributions (representatively shown for B4-doped Ge QD in Fig. 4). Since the spin splitting of the deep energy levels is associated with the polarizability of bonds, ${ }^{45}$ as the B atom moves from the isotropic configuration of B1 to the anisotropic configuration of B4, the spin splitting of the deep energy levels slightly increases from 78 to $107 \mathrm{meV}$. When a B atom is incorporated in the configuration of $\mathrm{B}_{\alpha}, \mathrm{B}_{\beta}$ or $\mathrm{B}_{\gamma}$, a deep energy level with empty electronic states is introduced near the conduction band of the Ge QD. The electron density of this energy level (LUMO) is mainly located at the B atom, while the electron density of the HOMO is dislocated among the Ge atoms

\section{HOMO}

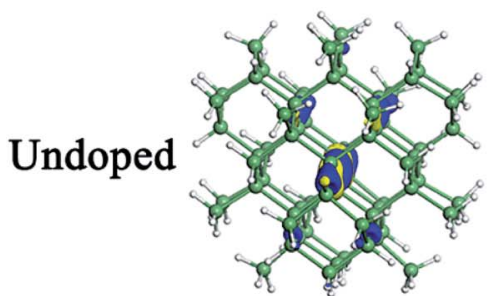

B4
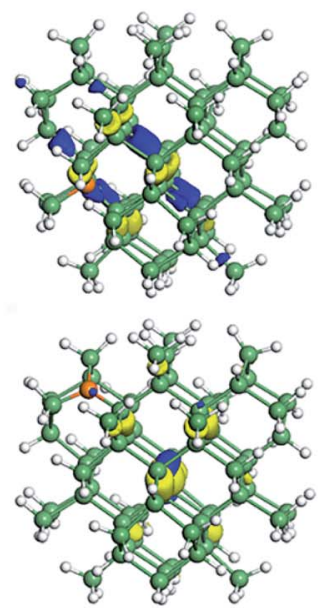

$\mathbf{B}_{\alpha}$
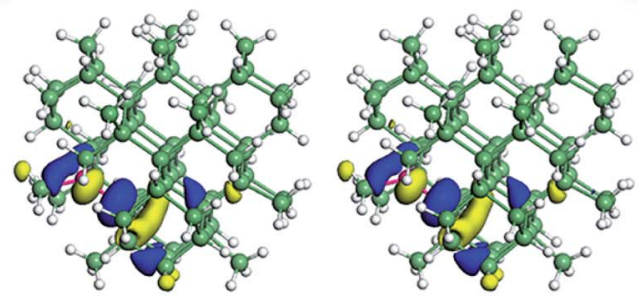

P4
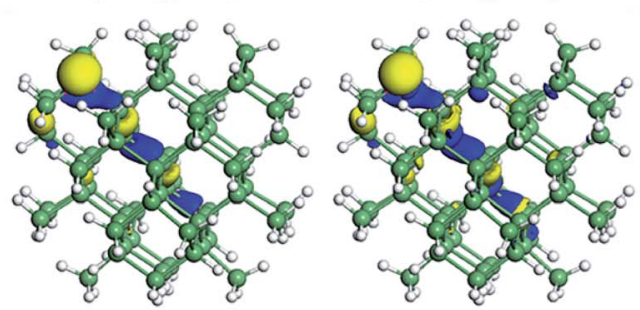

Fig. 4 Distribution of the isosurface for the electron density of the highest occupied molecular orbital (HOMO) and lowest unoccupied molecular orbital (LUMO) for undoped, B4-, B $\alpha^{-}$, P4- and $\mathrm{P}_{\alpha}$-doped Ge QDs. 
(representatively shown for $\mathrm{B}_{\alpha}$-doped Ge QD in Fig. 4). Similar to B-doped H-passivated Si QDs with B atoms at the QD surface, ${ }^{44}$ these deep energy levels are induced by the empty $2 \mathrm{p}_{z}$ orbitals of B. Since an empty $2 \mathrm{p}_{z}$ orbital is a good electron-acceptor, the electron cloud of an unconnected nearest-neighboring atom may shift to the empty $2 \mathrm{p}_{z}$ orbital of the B atom. This makes Bdoping-induced energy levels to move close to the conduction band of a Ge QD.

For P-doped Ge QDs, deep energy levels are introduced into the bandgap when P is in the configurations of P1, P2, P3 and $\mathrm{P} 4$. The P-doping-induced deep energy levels are close to the conduction band with a spin-splitting, proving the n-type characteristic of P-doped Ge QDs. This is caused by an extra electron induced by P doping in a Ge QD. In these cases, the electron densities of the HOMO and LUMO (deep energy levels) are mainly located at the $\mathrm{P}$ and neighboring Ge atoms with similar distributions (representatively shown for P4-doped Ge $\mathrm{QD}$ in Fig. 4). As a P atom moves from the center (P1) to the subsurface (P4) of the Ge QD, the deep energy levels step deeper into the middle of the bandgap while the spin splitting gradually increases from 88 to $280 \mathrm{meV}$. The former is due to the enhanced structural distortion induced by $\mathrm{P}$ doping from $\mathrm{P} 1$ to P4 (e.g., the bond length of a Ge-P bond is compressed by $15.9 \%$ and that of the adjacent Ge-P bond is stretched by $4.6 \%$ ) that causes a more localized electron density distribution of the deep energy levels, while the latter is due to the anisotropic transition of the polarizability of the Ge-P bonds from P1 to P4. We observe that the P-doping-induced energy levels are deeper than those induced by B doping, which is analogous to the results obtained on the doping of $\mathrm{B}$ and $\mathrm{P}$ in both hydrogenpassivated Si QDs ${ }^{43,44}$ and $\mathrm{Si}$ QDs that embedded in a $\mathrm{SiO}_{2}$ matrix. ${ }^{45,46}$ This infers the similarity of the electronic structures between Ge and Si QDs after B and P doping. When a P atom is incorporated in the configuration of $\mathrm{P}_{\alpha}, \mathrm{P}_{\beta}$ or $\mathrm{B}_{\gamma}$, no deep energy levels are introduced into the bandgap due to the threefold coordination of the electrons. The electron densities of both the HOMO and LUMO are located around P and neighboring Ge atoms. Again, this is similar to P-doped Si QDs. ${ }^{43}$

\section{B/P codoping}

We further investigate the effect of $\mathrm{B} / \mathrm{P}$ codoping for Ge QDs. As discussed above, a B (P) atom at the surface of a Ge QD does not act as an effective acceptor (donor). Thus, we only focus on the codoping of $\mathrm{B}$ and $\mathrm{P}$ when they are both incorporated inside a Ge QD. Under this condition, the configurations in which B and $\mathrm{P}$ are both incorporated in their energetically favored locations of 4 at the sub-surface of the QD are first considered. Depending on the distance between the $\mathrm{B}$ and $\mathrm{P}$ atoms, there exist three types of $\mathrm{B} / \mathrm{P}$-codoping configurations when both of them are doped at the locations of 4 . As the B-P distance increases, these three types of $\mathrm{B} / \mathrm{P}$-codoping configurations are denoted as $\mathrm{B} 4-\mathrm{P} 4{ }^{\prime}, \mathrm{B} 4-\mathrm{P} 4{ }^{\prime \prime}$ and $\mathrm{B} 4-\mathrm{P} 4{ }^{\prime \prime \prime}$. To figure out the influence of a dipole (i.e., a B-P pair) on the electronic properties of a Ge QD, we have considered the configurations in which $\mathrm{B}$ and $\mathrm{P}$ form a B-P pair, namely B3-P4 and B4-P3. Fig. 5 shows the models of (a) B4-P4" and (b) B4-P3 codoped Ge QDs.
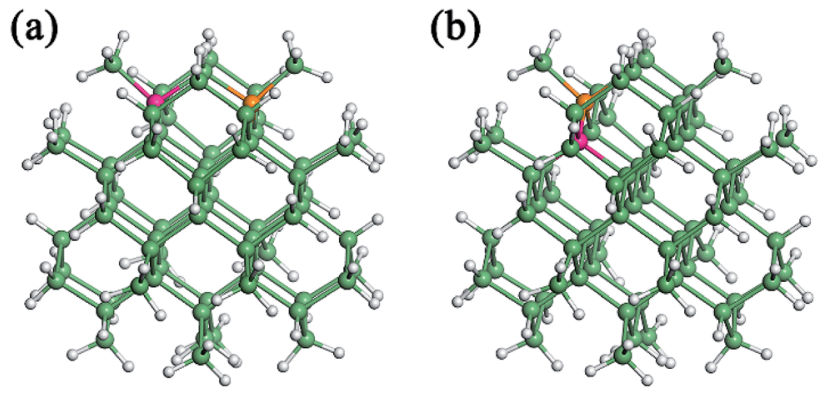

Fig. 5 Model of a Ge QD codoped with (a) B4-P4" or (b) B4-P3. Ge, $\mathrm{H}, \mathrm{B}$ and $\mathrm{P}$ atoms are denoted by green, grey, orange and pink balls, respectively.

The $E_{\mathrm{f}}$ of the dopants in a Ge QD is calculated by using ${ }^{56,57}$

$$
\begin{aligned}
E_{\mathrm{f}}=E\left(\mathrm{Ge}_{x^{\prime}} \mathrm{H}_{y^{\prime}} \mathrm{BP}\right) & -E\left(\mathrm{Ge}_{x} \mathrm{H}_{y}\right)-\mu_{\mathrm{B}}-\mu_{\mathrm{P}} \\
& -\left(x^{\prime}-x\right) \mu_{\mathrm{Ge}}-\left(y^{\prime}-y\right) \mu_{\mathrm{H}},
\end{aligned}
$$

where $E\left(\mathrm{Ge}_{x^{\prime}} \mathrm{H}_{y^{\prime}} \mathrm{BP}\right)$ is the total energy of a Ge QD after the codoping of B and P. The calculated $E_{\mathrm{f}}$ of B/P dopants in a Ge

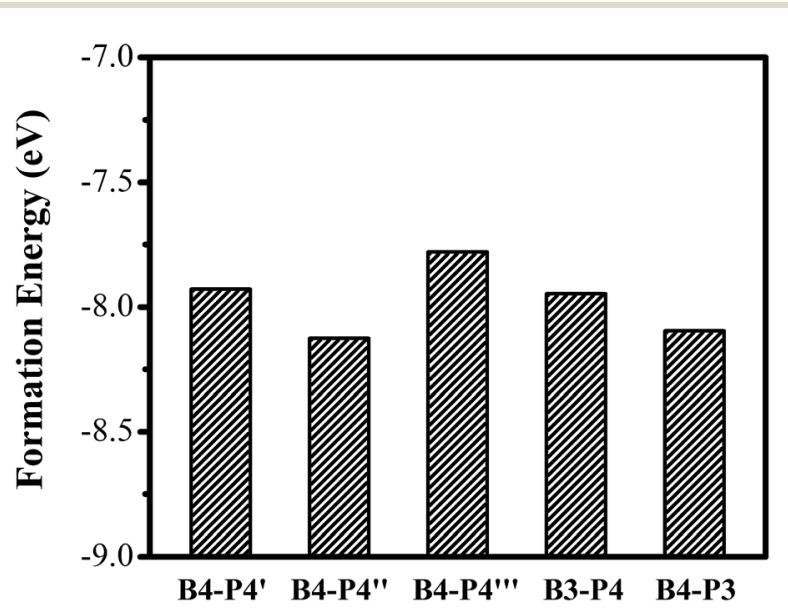

Fig. 6 Formation energy of B/P dopants in a Ge QD.

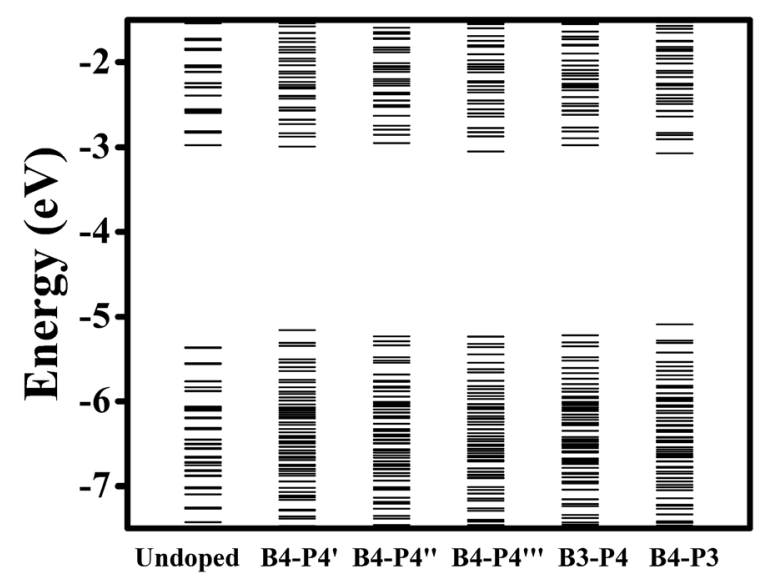

Fig. 7 Energy-level diagrams for undoped and B/P-codoped Ge QDs at the ground state. 
QD is shown in Fig. 6. It is seen that the values of $E_{\mathrm{f}}$ for $\mathrm{B} / \mathrm{P}$ dopants is in the range of -8.1 to $-7.8 \mathrm{eV}$, which is much smaller than that for a singular B or P atom in a Ge QD. This indicates that the codoping of $\mathrm{B}$ and $\mathrm{P}$ may actually facilitate the incorporation of $\mathrm{B}$ and $\mathrm{P}$ into Ge QDs. In contrast to a $\mathrm{B} / \mathrm{P}$

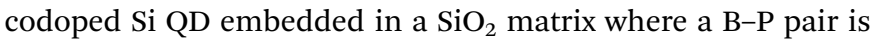
the most likely to be formed in the QD, ${ }^{\mathbf{4 6}}$ the $E_{\mathrm{f}}$ of a B-P pair is basically the same as that of unpaired B or P atom in a Ge QD. This implies that a B atom and a $\mathrm{P}$ atom would be randomly distributed at the sub-surface of a Ge QD when they are codoped into the QD.

The energy-level diagrams for undoped and B/P codoped Ge QDs are shown in Fig. 7. Similar to B/P codoped H-passivated Si $\mathrm{QDs},{ }^{63} \mathrm{~B} / \mathrm{P}$ codoping reduces the bandgaps of Ge QDs by $0.11-$

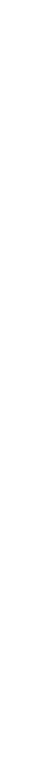

B4-P4'
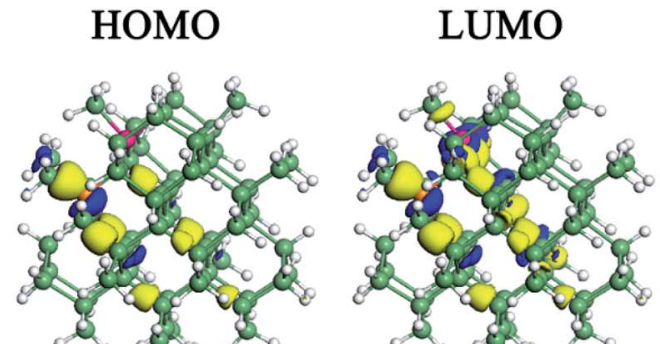

B4-P4"
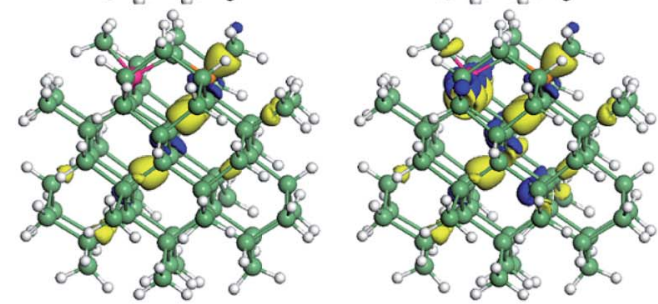

B4-P4"'
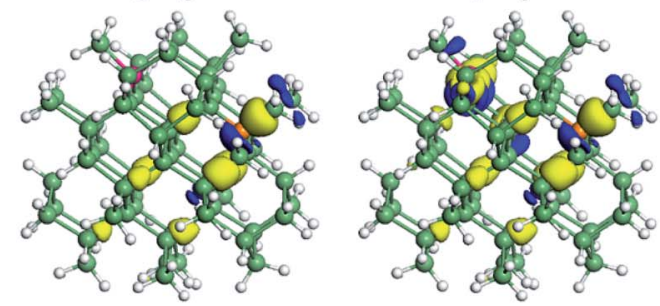

B3-P4
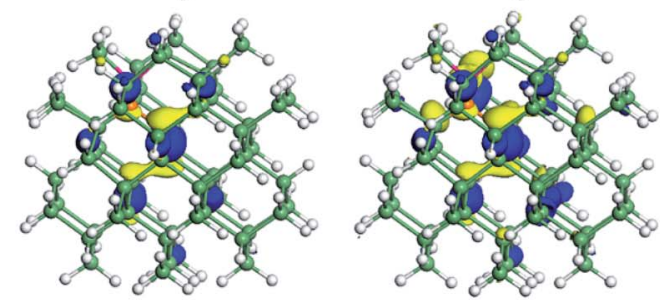

B4-P3
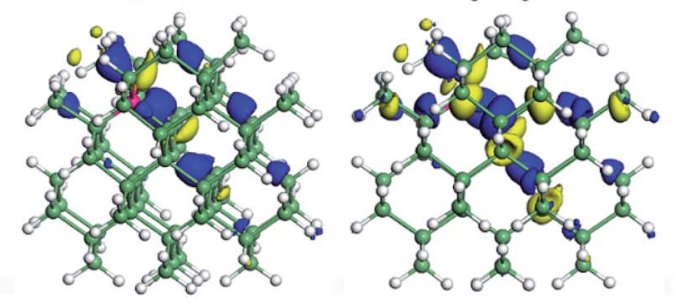

Fig. 8 Distribution of the isosurface for the electron density of the highest occupied molecular orbital (HOMO) and lowest unoccupied molecular orbital (LUMO) for B/P-codoped Ge QDs.
$0.37 \mathrm{eV}$. This highlights the tunability of band structures of Ge QDs upon $\mathrm{B} / \mathrm{P}$ codoping. The reduction of the bandgaps of $\mathrm{Ge}$ QDs is mainly due to the rise of the HOMO levels towards the middle of the bandgaps (Fig. 7). For B/P codoped Ge QDs, the electron densities of the HOMO are localized at the $\mathrm{B}$ and neighboring Ge atoms, while those of the LUMO are distributed around $\mathrm{B}, \mathrm{P}$ and $\mathrm{Ge}$ atoms (Fig. 8). It is the localization of the electron density of the HOMO on B that contributes to the uplift of the HOMO level. It is interesting that the formation of a B-P pair does not distinctly affect the band structures of $\mathrm{B} / \mathrm{P}$ codoped Ge QDs. This is again quite different from Si QDs embedded in $\mathrm{a} \mathrm{SiO}_{2}$ matrix, in which a $\mathrm{B}-\mathrm{P}$ pair alters the band structure of codoped Si QDs by forming a static electric dipole that radially points toward the QD core. ${ }^{46}$

\section{Conclusions}

We have studied the doping of Ge QDs with B and P by means of density functional theory. The current results show that $\mathrm{B}$ or $\mathrm{P}$ is the most likely incorporated at the near-surface region of $\mathrm{Ge}$ QDs. B doping introduces deep energy levels in the bandgap of a Ge QD whether it is inside or at the surface of the QD. In contrast, $\mathrm{P}$ doping causes deep energy levels only when it is incorporated inside the Ge QD. The codoping of B and P may promote the incorporation of $\mathrm{B}$ and $\mathrm{P}$ into Ge QDs, given a lower formation energy of $\mathrm{B} / \mathrm{P}$ dopants. After $\mathrm{B} / \mathrm{P}$ codoping, the bandgap of a Ge QD is reduced. Considering the moderate bandgaps ( $\sim 2.02$ to $2.52 \mathrm{eV}$ ) and suitable band alignment with water, B- and P-doped Ge QDs may have the potential applications in photocatalytic water splitting. ${ }^{64}$ Our calculated results inspire the modulation of the band structures of Ge QDs for desirable applications by $\mathrm{B}$ and $\mathrm{P}$ doping in the future.

\section{Conflicts of interest}

There are no conflicts to declare.

\section{Acknowledgements}

Shanghai Supercomputer Center is acknowledged for providing computation resources. This study is mainly supported by the National Key Research and Development Program of China (Grant No. 2017YFA0205700), Natural Science Foundation of China (NSFC) (Grant No. 61774133 and 6147409), NSFC-FWO program (Grant No. 61411130229 and 61511130264) and Program for Innovative Research Team in University of Ministry of Education of China (IRT13R54).

\section{References}

1 J. Zhu and M. C. Hersam, Adv. Mater., 2017, 29, 1603895.

2 D. K. Kim, Y. Lai, B. T. Diroll, C. B. Murray and C. R. Kagan, Nat. Commun., 2012, 3, 1216.

3 Q. B. Luan, Z. Y. Ni, S. Koura, T. J. Zhu, D. Yang and X. D. Pi, AIP Adv., 2014, 4, 127108. 
4 M.-S. Yeh, Y.-C. Wu, M.-H. Chung, Y.-R. Jhan, K.-S. ChangLiao, K.-C. Liu, M.-H. Wu and M.-F. Hung, Appl. Phys. Lett., 2014, 105, 042109.

5 M. Yuan, M. Liu and E. H. Sargent, Nat. Energy, 2016, 1, 16016.

6 S. Y. Zhao, X. D. Pi, C. Mercier, Z. C. Yuan, B. Q. Sun and D. Yang, Nano Energy, 2016, 26, 305-312.

7 X. D. Pi, L. Zhang and D. R. Yang, J. Phys. Chem. C, 2012, 116, 21240-21243.

8 J. Xu, S. H. Sun, Y. Q. Cao, P. Lu, W. Li and K. J. Chen, Part. Part. Syst. Charact., 2014, 31, 459-464.

9 Z. Y. Ni, L. L. Ma, S. C. Du, Y. Xu, M. Yuan, H. H. Fang, Z. Wang, M. S. Xu, D. S. Li, J. Y. Yang, W. D. Hu, X. D. Pi and D. Yang, ACS Nano, 2017, 11, 9854-9862.

10 V. Adinolfi and E. H. Sargent, Nature, 2017, 542, 324-327.

11 T. Yu, F. Wang, Y. Xu, L. L. Ma, X. D. Pi and D. Yang, Adv. Mater., 2016, 28, 4912-4919.

12 F. P. García de Arquer, A. Armin, P. Meredith and E. H. Sargent, Nat. Rev. Mater., 2017, 2, 16100.

13 L. Yao, T. Yu, L. X. Ba, H. Meng, X. Fang, Y. L. Wang, L. Li, X. Rong, S. Wang, X. Q. Wang, G. Z. Ran, X. D. Pi and G. G. Qin, J. Mater. Chem. C, 2016, 4, 673-677.

14 F. Maier-Flaig, J. Rinck, M. Stephan, T. Bocksrocker, M. Bruns, C. Kubel, A. K. Powell, G. A. Ozin and U. Lemmer, Nano Lett., 2013, 13, 475-480.

15 W. Gu, X. K. Liu, X. D. Pi, X. L. Dai, S. Y. Zhao, L. Yao, D. S. Li, Y. Z. Jin, M. S. Xu, D. Yang and G. G. Qin, IEEE Photonics J., 2017, 9, 1-10.

16 X. Cheng, S. B. Lowe, P. J. Reece and J. J. Gooding, Chem. Soc. Rev., 2014, 43, 2680-2700.

17 Y. L. Zhong, X. T. Sun, S. Y. Wang, F. Peng, F. Bao, Y. Y. Su, Y. Y. Li, S.-T. Lee and Y. He, ACS Nano, 2015, 9, 5958-5967.

18 J. Kang, J. Joo, E. J. Kwon, M. Skalak, S. Hussain, Z. G. She, E. Ruoslahti, S. N. Bhatia and M. J. Sailor, Adv. Mater., 2016, 28, 7962-7969.

19 L. Jing, S. V. Kershaw, Y. Li, X. Huang, Y. Li, A. L. Rogach and M. Gao, Chem. Rev., 2016, 116, 10623-10730.

20 C. R. Kagan and C. B. Murray, Nat. Nanotechnol., 2015, 10, 1013.

21 Z. Y. Ni, X. D. Pi, M. Ali, S. Zhou, T. Nozaki and D. R. Yang, J. Phys. D: Appl. Phys., 2015, 48, 314006.

22 D. Mocatta, G. Cohen, J. Schattner, O. Millo, E. Rabani and U. Banin, Science, 2011, 332, 77-81.

23 A. Sahu, M. S. Kang, A. Kompch, C. Notthoff, A. W. Wills, D. Deng, M. Winterer, C. D. Frisbie and D. J. Norris, Nano Lett., 2012, 12, 2587-2594.

24 B. L. Greenberg, S. Ganguly, J. T. Held, N. J. Kramer, K. A. Mkhoyan, E. S. Aydil and U. R. Kortshagen, Nano Lett., 2015, 15, 8162-8169.

25 M. Fujii, H. Sugimoto and K. Imakita, Nanotechnology, 2016, 27, 262001.

26 P. Lu, W. Mu, J. Xu, X. Zhang, W. Zhang, W. Li, L. Xu and K. Chen, Sci. Rep., 2016, 6, 22888.

27 J. M. Luther, P. K. Jain, T. Ewers and A. P. Alivisatos, Nat. Mater., 2011, 10, 361-366.

28 S. W. Hsu, K. On and A. R. Tao, J. Am. Chem. Soc., 2011, 133, 19072-19075.
29 S. Zhou, X. D. Pi, Z. Y. Ni, Y. Ding, Y. Y. Jiang, C. H. Jin, C. Delerue, D. R. Yang and T. Nozaki, ACS Nano, 2015, 9, 378-386.

30 S. Zhou, Z. Y. Ni, Y. Ding, M. Sugaya, X. D. Pi and T. Nozaki, ACS Photonics, 2016, 3, 415-422.

31 Z. Y. Ni, X. D. Pi, S. Zhou, T. Nozaki, B. Grandidier and D. Yang, Adv. Opt. Mater., 2016, 4, 700-707.

32 X. D. Pi and C. Delerue, Phys. Rev. Lett., 2013, 111, 177402.

33 M. Kanehara, H. Koike, T. Yoshinaga and T. Teranishi, J. Am. Chem. Soc., 2009, 131, 17736-17737.

34 A. R. Stegner, R. N. Pereira, K. Klein, R. Lechner, R. Dietmueller, M. S. Brandt, M. Stutzmann and H. Wiggers, Phys. Rev. Lett., 2008, 100, 026803.

35 R. Gresback, N. J. Kramer, Y. Ding, T. Chen, U. R. Kortshagen and T. Nozaki, ACS Nano, 2014, 8, 5650-5656.

36 D. A. Ruddy and N. R. Neale, ACS National Meeting, 2012, INOR-1288.

37 L. Men, M. A. White, H. Andaraarachchi, B. A. Rosales and J. Vela, Chem. Mater., 2017, 29, 168-175.

38 B. W. Boote, L. Men, H. P. Andaraarachchi, U. Bhattacharjee, J. W. Petrich, J. Vela and E. A. Smith, Chem. Mater., 2017, 29, 6012-6021.

39 K. Ramasamy, P. G. Kotula, A. F. Fidler, M. T. Brumbach, J. M. Pietryga and S. A. Ivanov, Chem. Mater., 2015, 27, 4640-4649.

40 R. J. A. Esteves, M. Q. Ho and I. U. Arachchige, Chem. Mater., 2015, 27, 1559-1568.

41 T. H. Yuan, X. D. Pi and D. Yang, IEEE J. Sel. Top. Quantum Electron., 2017, 23, 4800205.

42 Y. Gao, X. D. Pi, X. H. Wang, T. H. Yuan, Q. J. Jiang, R. Gresback, J. G. Lu and D. Yang, Part. Part. Syst. Charact., 2016, 33, 271-278.

43 X. B. Chen, X. D. Pi and D. Yang, J. Phys. Chem. C, 2011, 115, 661-666.

44 X. D. Pi, X. B. Chen and D. Yang, J. Phys. Chem. C, 2011, 115, 9838-9843.

45 Z. Y. Ni, X. D. Pi and D. Yang, Phys. Rev. B: Condens. Matter Mater. Phys., 2014, 89, 035312.

46 Z. Y. Ni, X. D. Pi, S. Cottenier and D. Yang, Phys. Rev. B, 2017, 95, 075307.

47 A. Puzder, A. Williamson, J. Grossman and G. Galli, Phys. Rev. Lett., 2002, 88, 097401.

48 L. Ramos, E. Degoli, G. Cantele, S. Ossicini, D. Ninno, J. Furthmüller and F. Bechstedt, Phys. Rev. B: Condens. Matter Mater. Phys., 2008, 78, 235310.

49 R. Guerra and S. Ossicini, J. Am. Chem. Soc., 2014, 136, 44044409.

50 X. D. Pi, R. Wang and D. Yang, Chin. Phys. B, 2014, 23, 076102. 51 B. Delley, J. Chem. Phys., 2000, 113, 7756.

52 X. Liu, Y. Zhang, T. Yu, X. Qiao, R. Gresback, X. Pi and D. Yang, Part. Part. Syst. Charact., 2016, 33, 44-52.

53 S. Niesar, R. N. Pereira, A. R. Stegner, N. Erhard, M. Hoeb, A. Baumer, H. Wiggers, M. S. Brandt and M. Stutzmann, Adv. Funct. Mater., 2012, 22, 1190-1198.

54 S. M. Pratik, A. Nijamudheen and A. Datta, Chemistry, 2015, 21, 18454-18460. 
55 A. Nijamudheen, R. Bhattacharjee, S. Choudhury and A. Datta, J. Phys. Chem. C, 2015, 119, 3802-3809.

56 E. Degoli, S. Ossicini, G. Cantele, E. Luppi, R. Magri, D. Ninno and O. Bisi, Phys. Status Solidi C, 2005, 2, 3354-3358.

57 A. Carvalho, S. Öberg, M. J. Rayson and P. R. Briddon, Phys. Rev. B: Condens. Matter Mater. Phys., 2012, 86, 045308.

58 L. G. Wang and A. Zunger, Phys. Rev. B: Condens. Matter Mater. Phys., 2002, 66, 161202.

59 X. D. Pi, J. Nanomater., 2012, 2012, 912903.
60 S. Zhou, X. D. Pi, Z. Y. Ni, Q. B. Luan, Y. Y. Jiang, C. H. Jin, T. Nozaki and D. R. Yang, Part. Part. Syst. Charact., 2015, 32, 213-221.

61 T. Teshome and A. Datta, J. Phys. Chem. C, 2017, 121, 1516915180.

62 E. G. Barbagiovanni, D. J. Lockwood, P. J. Simpson and L. V. Goncharova, J. Appl. Phys., 2012, 111, 034307.

63 Y. S. Ma, X. B. Chen, X. D. Pi and D. Yang, J. Nanopart. Res., 2012, 14, 802.

64 C. Chowdhury, S. Karmakar and A. Datta, J. Phys. Chem. C, 2017, 121, 7615-7624. 